\title{
Relative motion in spacetime
}

\author{
Philippe Droz-Vincent \\ $L U T H^{*}$
}

\begin{abstract}
In Minkowski spacetime, we consider an isolated system made of two pointlike bodies interacting at a distance in the nonradiative approximation. Our framework is the covariant and a priori Hamiltonian formalism of "predictive relativistic mechanics", founded on the equal-time condition. The issue of an equivalent one-body description is discussed. We distinguish two different concepts: on the one hand an almost purely kinematic relative particle, on the other hand an effective particle which involves an explicit dynamical formulation; several versions of the latter are possible. Relative and effective particles have the same orbit, but may differ by their schedules.
\end{abstract}

\section{Introduction}

The concept of relative motion is basically kinematic; it rests upon the notion of radius-vector defined as the difference of the positions, it is viewed as the motion of a ficticious particle referred to as the "relative particle". In nonrelativistic mechanics the two-body problem (concerning an isolated system) is easily reduced to a one-body problem concerning the relative particle (affected by the "reduced mass"). Of course, in order to be equivalent to the binary motion, the relative motion must be considered together with that of the center of mass.

In the framework of special relativity the situation becomes more complicated. On the one hand extending to spacetime the construction of relative particle with help of a radius-vector is natural and straightforward, provided we have a center of mass at our disposal. On the

*Observatoire de Paris-Meudon, CNRS, Université Paris Diderot, 5 place Jules Janssen, 92195 Meudon, France 
other hand it remains to be checked how the result of this procedure is actually equivalent to the binary motion.

In fact the need of considering an equivalent one-body motion first arose in the realm of relativistic quantum mechanics: in an early attempt by Itzykson et al. a relativistic reduced mass was suggested [1]. Todorov, elaborating his quasi-potential approach to the two-body problem [2], systematically invoked an effective particle supposed to satisfy a Klein-Gordon equation (with external field). The classical relativistic version of his work [3] was inspired from Dirac's constraint theory [4]; there again, the task of solving the equations of the binary system was reduced to an effective one-body problem.

More recently these topics were considered in the framework of the "restframe instant form" of relativistic dynamics [5], but due to a different definition of center of mass, the contact of our approach with that work would be far from straightforward.

For several authors the interest in relative motion was aiming at an approximate treatment of the gravitational two-body problem in General Relativity [6] [7] [8], see also [9], but the scope of the present paper is strictly limited to Minkowski spacetime and special relativity. We have in mind the two-body conservative dynamics of directly interacting point particles; the field carrying interaction is supposed to be eliminated. Following the a priori Hamiltonian approach [10] 11], we shall use the manifestly covariant formalism of predictive relativistic mechanics [12] [13, in the version where positions are defined by an equal-time condition; this point of view offers several advantages:

1) it can be re-formulated in terms of constraints, as shown in detail by L. Lusanna [14].

2) the relative orbit lays in a two-dimensional (spacelike) plane.

3) it allows for a covariant definition of center of mass [15] which has the physical meaning of Pryce's definition [16].

Provided the existance of a linearly moving center of mass is assumed from the outset, the relative worldline always can be (in principle) constructed without difficulty. In this procedure the input is a two-body motion, that is a pair of worldlines, and the output is a one-body motion, that is a single worldline; at this stage we do not yet consider systems.

Somehow different is the concept of "equivalent" (or "effective") onebody system. Given a binary system (defined by equations of motion derived from a Hamiltonian formalism) one aims at finding solutions, and one hopes to reduce this problem to the solving of a single-body problem. One would expect that the relative particle defined on kine- 
matic grounds is apropriate for this task. But investigating under which circumstances this guess is correct one runs into severe conditions; therefore we shall distinguish "relative particle" from "equivalent one-body" system, keeping in mind however that, for the sake of simplicity and efficiency, the latter should depart from the former as little as possible.

Section 2 is a summary of the covariant Hamiltonian formalism we use for one-body and two-body systems. Relative motion is analyzed in Section 3, and Section 4 is devoted to concluding remarks.

Terminology, notation Units $c=\hbar=1$. Greek indices $0,1,2,3$. Latin indices $1,2,3$. Indicator $\varepsilon^{0123}=1$. When no confusion is possible tensor indices are omitted, and the contraction dot is employed also for tensors, so for instance $J \cdot P$ stands for $J^{\alpha \mu} P_{\mu}$. Pointlike bodies are "particles"; particle labels are $a, b$.

\section{One-body and two-body systems}

The motion of a single pointlike body of coordinates $x^{\alpha}$ is represented by its worldline. An inertial observer (of unspecified mass) is characterized by a timelike straight line of direction $u^{\alpha}$. Taking the origin of coordinates anywhere on the observer's worldline, the orbit of the body is, strictly speaking, the set of all the spacelike four-vectors $x_{\perp}^{\alpha}$, where $x_{\perp}=\delta_{\perp} x$, using the projector

$$
\delta_{\perp \beta}^{\alpha}=\delta_{\beta}^{\alpha}-u^{\alpha} u_{\beta}
$$

But naturally the orbit can be trivially identified with the orthogonal projection of the body's worldline onto any three-plane orthogonal to $u$. The schedule of the body consists in a relation between the points of the orbit and the observer's time.

\subsection{One body in a stationary external potential}

Now consider a single pointlike body submitted to a stationary external potential generated by a massive source at rest; this source is a distinguished inertial observer. Using the Poisson brackets

$$
\left(x^{\alpha}, p_{\beta}\right)=\delta_{\beta}^{\alpha}
$$

we write the canonical equations of motion in terms of some evolution parameter $\tau$ ( most often but not always, this parameter is affine; for instance it is not when the external field is a weak gravitational one)

$$
\frac{d x}{d \tau}=(x, H), \quad \frac{d p}{d \tau}=(p, H)
$$


The Hamiltonian generator

$$
H=\frac{1}{2} p^{2}+W
$$

is a constant of motion numerically identified with the half-squared mass. $W$ is a scalar referred to as the "potential".

Remark In our unconstrained eight-dimensional phase space, mass is not specified a priori, rather it appears as a constant of the motion; in this framework the dynamical system encompasses all the possible numerical values of mass.

For our purpose it is convenient to separate space from time, with help of the projector $\delta_{\perp}$. The assumption of stationarity means that $W$ is a function of only $x_{\perp}, p_{\perp}, p_{0}$. In any frame adapted to $u$ we have that

$$
\left(x_{\perp}, p_{0}\right)=\left(p_{\perp}, p_{0}\right)=0
$$

so it is clear that the spatial piece of the canonical equations of motion can be written as well

$$
\begin{aligned}
\frac{d}{d \tau} x_{\perp}^{\alpha} & =\left(x_{\perp}^{\alpha}, \frac{1}{2} p_{\perp}^{2}+W\right) \\
\frac{d}{d \tau} p_{\perp}^{\alpha} & =\left(p_{\perp}^{\alpha}, \frac{1}{2} p_{\perp}^{2}+W\right)
\end{aligned}
$$

Remark Since $\left(x_{\perp}^{\alpha}, p_{0}\right)=0$ we could replace $p_{\perp}^{2}$ by $p^{2}$ in the r.h.s. of (4) (5).

The timelike piece of the canonical equations of motion consists in

$$
\begin{gathered}
\frac{d p_{0}}{d \tau}=0 \\
\frac{d x_{0}}{d \tau}=p_{0}+\frac{\partial W}{\partial p_{0}}
\end{gathered}
$$

hence

$$
p_{0}=E=\text { const. }
$$

which can be inserted into system (4) (15).

Let us solve the spatial system (4) (5) above. Since $x_{\perp}$ and $p_{\perp}$ have vanishing Poisson brackets with $p_{0}$, this quantity can be treated as a constant, say $p_{0}=E$, when computing the r.h.s. in (4) (5). The task of integrating these equationsis similar to a nonrelativistic and threedimensional problem. Beside initial data, its solution depends on $E$ as a parameter, say

$$
x_{\perp}=\mathcal{X}_{\perp}(\tau, E), \quad p_{\perp}=\mathcal{P}_{\perp}(\tau, E)
$$


Finally we integrate eqn (17)

$$
x^{0}=E \tau+\int \frac{\partial W}{\partial p_{0}} d \tau
$$

where the integrand is considered as a function of $\tau$ through (9) and $E$ behaves as a parameter. We can summarize:

Proposition 1 In any adapted frame, the coordinate time of the body, namely $x^{0}$ is always the proper time (and also the coordinate time) of the source, whatever is the mass of the source.

In the simple case where $\partial W / \partial p_{0}$ is identically zero 1 , and using an adapted frame, $x^{0}$ is proportional to the evolution parameter; in this case $E$ is necessarily positive.

Since the potential is stationary, energy $E$ (evaluated in the frame of the source) is conserved, see (8). Since $2 H=m^{2}$ we have

$$
p_{\perp}^{2}+2 W=m^{2}-E^{2}
$$

Setting $p_{\perp}^{2}=-\mathbf{p}^{2}$ we can introduce the nonrelativistic energy $E_{\mathrm{NR}}=$ $\frac{\mathbf{p}^{2}}{2 m}-\frac{W}{m}$ which is obviously a constant of the motion, and we can rigorously write

$$
E^{2}=m^{2}+2 m E_{\mathrm{NR}}
$$

so the well-known formula $E=m+E_{\mathrm{NR}}+\cdots$ is obtained as a development in powers of $\frac{E_{\mathrm{NR}}}{m}$ (and the nonrelativistic limit corresponds to $\left.E_{\mathrm{NR}} \ll m\right)$.

Rest energy simply is $m$, so we can define unrest energy as $E-m$.

Remark Let a worldline be defined by

$$
x^{i}=f^{i}(\tau), \quad x^{0}=g(\tau)
$$

and let $\bar{g}$ be the inverse function of $g$, say $\tau=\bar{g}\left(x^{0}\right)$. Finally the worldline is as well defined by

$$
x^{i}=f^{i}\left(\bar{g}\left(x^{0}\right)\right)
$$

Notice that if in (12) we change only the second formula (in other words we modify $g(\tau)$, leaving $f^{i}$ unchanged) then we keep the same orbit but the schedule is modified, and of course the worldline gets changed.

\footnotetext{
${ }^{1}$ for instance the toy model presented in 17
} 


\subsection{Two-body isolated system}

The reader is referred to [1] [17] for details. The Hamiltonian generators of motion are

$$
H_{1}=\frac{1}{2} p_{1}^{2}+V_{1}, \quad H_{2}=\frac{1}{2} p_{2}^{2}+V_{2}
$$

The interaction potentials $V_{a}$ are functions on a 16-dimensional phase space and the squared masses appear as first integrals, say $2 H_{a}=m_{a}^{2}$. The canonical variables $q_{a}^{\alpha}, p_{b}^{\beta}$ are submitted to standard Poisson brackets $\left\{q_{a}^{\alpha}, p_{b \beta}\right\}=\delta_{a b} \delta_{\beta}^{\alpha}$. Due to a famous no-go theorem [18], the canonical coordinates $q_{a}^{\alpha}$ cannot coincide on the whole phase space with the physical positions $x_{a}^{\alpha}$.

Notation Collective and relative canonical variables are respectively

$$
\begin{gathered}
P=p_{1}+p_{2}, \quad Q=\frac{1}{2}\left(q_{1}+q_{2}\right) \\
y=\frac{1}{2}\left(p_{1}-p_{2}\right) \quad z=q_{1}-q_{2}
\end{gathered}
$$

The system being isolated, the interaction potentials $V_{a}$ are Poincaré invariant. Poincaré algebra is generated by linear momentum $P$ and by angular momentum

$$
J=q_{1} \wedge p_{1}+q_{2} \wedge p_{2}=Q \wedge P+z \wedge y
$$

The relative physical position

$$
r=x_{1}-x_{2}
$$

should not be confused with $z$, except at equal times.

Masses are $m_{1}, m_{2}$. It is convenient to set

$$
\mu=\frac{1}{2}\left(m_{1}^{2}+m_{2}^{2}\right), \quad \nu=\frac{1}{2}\left(m_{1}^{2}-m_{2}^{2}\right)
$$

Without loss of generality we assume $m_{1} \leq m_{2}$. Let $m_{0}=m_{1} m_{2} /\left(m_{1}+m_{2}\right)$ be the usual nonrelativistic reduced mass; hence the inequalities

$$
\frac{1}{4} m_{1}^{2} \leq m_{0}^{2}<m_{1}^{2} \leq \mu \leq m_{2}^{2}
$$

note that $m_{1}$ and $m_{0}$ keep the same order of magnitude. The nonrelativistic approximation is characterized by neglecting $|\Lambda|$ in front of both $m_{1}^{2}$ and $m_{2}^{2}$. Post-Galilean developments can be carried out as expansions in powers of the dimensionless quantity $\Lambda / m_{0}^{2}$. 
The canonical equations of motion are

$$
\frac{\partial q_{a}}{\partial \tau_{b}}=\left\{q_{a}, \frac{1}{2} p_{b}^{2}+V_{b}\right\}
$$

in terms of individual evolution parameters $\tau_{1}, \tau_{2}$ (generally not affine). We define

$$
V=\frac{1}{2}\left(V_{1}+V_{2}\right), \quad U=\frac{1}{2}\left(V_{1}-V_{2}\right)
$$

Poincare invariance of both $V_{1}$ and $V_{2}$ entails that $P^{\alpha}$ and $J_{\mu \nu}$ are constants of the motion.

The coordinates of the center of mass are

$$
\Xi=Q+\left(\frac{y \cdot P}{P^{2}}\right) z-\left(\frac{z \cdot P}{P^{2}}\right) y
$$

thus we have throughout phase space

$$
\Xi \cdot P=Q \cdot P
$$

Formula (16) admits two alternative and equivalent expressions, namely

$$
\Xi=\frac{P \cdot p_{1}}{P^{2}} q_{1}+\frac{P \cdot p_{2}}{P^{2}} q_{2}+\frac{P \cdot z}{P^{2}} y
$$

which involves the individual energies

$$
M_{1}=\frac{P \cdot p_{1}}{\sqrt{P^{2}}}, \quad M_{2}=\frac{P \cdot p_{2}}{\sqrt{P^{2}}}
$$

and also

$$
\Xi=\frac{J \cdot P}{P^{2}}+\left(\frac{P \cdot Q}{P^{2}}\right) P
$$

with notation $(J \cdot P)^{\alpha}=J^{\alpha \beta} P_{\beta}$, thus defining

$$
T=\frac{P \cdot Q}{|P|}
$$

it turns out that $T$ is the proper time of the center of mass 2

Fixing the total linear momentum (say $P^{\alpha}=k^{\alpha}$ with $k \cdot k=M^{2}$ ) defines the rest frame, where $\Xi^{0}=T+$ const. We shall always take the origin of coordinates on the worldline of the center of mass, therefore

$$
\Xi^{0}=T
$$

\footnotetext{
${ }^{2} \mathrm{~T}$ has dimension of time, in contrast to the evolutions parameters $\tau_{1}, \tau_{2}$.
} 
Notation

$$
\begin{aligned}
& \Pi_{\beta}^{\alpha}=\delta_{\beta}^{\alpha}-P^{\alpha} P_{\beta} / P \cdot P, \quad \widehat{\delta}_{\beta}^{\alpha}=\delta_{\beta}^{\alpha}-k^{\alpha} k_{\beta} / k \cdot k \\
& \widetilde{x}=\Pi x, \quad \widehat{x}=\widehat{\delta} x, \text { etc. }
\end{aligned}
$$

Consider the equal-time description; the equal-time manifold included in phase space is defined by $P \cdot z=0$.

In the rest frame:

$z^{0}=0$ in other words $q_{1}^{0}=q_{2}^{0}$, thus $x_{1}^{0}=x_{2}^{0}$. On the other hand

$$
\Xi^{0}=Q^{0}=\frac{1}{2}\left(q_{1}^{0}+q_{2}^{0}\right)=\frac{1}{2}\left(x_{1}^{0}+x_{2}^{0}\right)
$$

hence $x_{1}^{0}=x_{2}^{0}=T$.

In general we have

$$
y \cdot P=\nu-2 U
$$

so finally the equal-time description takes on this form

$$
\begin{gathered}
\widehat{x}_{1}=\widehat{\Xi}+\left(\frac{2 U-\nu}{M^{2}}+\frac{1}{2}\right) r \\
\widehat{x}_{2}=\widehat{\Xi}+\left(\frac{2 U-\nu}{M^{2}}-\frac{1}{2}\right) r \\
x_{1}^{0}=T, \quad x_{2}^{0}=T
\end{gathered}
$$

Definition The orbit of a two-body motion is the set of values taken by $\widehat{r}$ in the three-dimensional vector space orthogonal to $k$.

Owing to the constancy of angular momentum, it is a plane curve included in the two-dimensional vector space orthogonal to linear momentum $P^{\alpha}$ and to the Pauli-Lubanski vector

$$
L_{\alpha}=\varepsilon_{\alpha \mu \nu \rho} P^{\mu} \widetilde{z}^{\nu} \widetilde{y}^{\rho}
$$

\subsection{Unipotential models}

Several simplifications arise when $U$ is identically zero on the whole phase space, in other words $V_{1}=V_{2}=V$. In this case

$$
V=V\left(\widetilde{z}^{2}, \widetilde{y}^{2}, \widetilde{z} \cdot \widetilde{y}, P^{2}, y \cdot P\right)
$$

and it automatically turns out that $y \cdot P$ is a constant of the motion. This particular model, referred to as unipotential, is still general enough 
for describing a lot of physical situations. In this case it is convenient to set

$$
\lambda=\tau_{1}+\tau_{2}
$$

so the equations of motion for $\widetilde{z}, \widetilde{y}$ can be written as [19]

$$
\begin{aligned}
& \frac{d \widetilde{z}}{d \lambda}=\left\{\widetilde{z}, \frac{1}{2} \widetilde{y}^{2}+V\right\} \\
& \frac{d \widetilde{y}}{d \lambda}=\left\{\widetilde{y}, \frac{1}{2} \widetilde{y}^{2}+V\right\}
\end{aligned}
$$

where the brackets can be computed as functions of $\widetilde{z}^{\mu}, \widetilde{y}^{\nu}$, and of the first integrals $P^{2}, y \cdot P$. Once $P^{2}$ and $y \cdot P$ have been fixed, the evolution of the spatial internal variables is thus given by a system of six firstorder differential equations, to solve for six unknown functions; this problem has the structure of a nonrelativistic problem for one body in three dimensions.

Remark: In the evolution equations above, $P^{2}$ and $y \cdot P$ have vanishing Poisson brackets with $\widetilde{y}^{2}+2 V$ (remember $\mathrm{V}$ doesnot depend on $z \cdot P$ ), thus they behave like constants when computing the right-hand sides. The four-vectors $\widetilde{z}$ and $\widetilde{y}$ remain within the 2-plane orthogonal to $k$ and to the (conserved) Pauli-Lubanski vector. Note this constant of the motion

$$
j^{2}=\widetilde{z}^{2} \widetilde{y}^{2}-(\widetilde{z} \cdot \widetilde{y})^{2}
$$

Let a solution of (31) (32) be of the form

$$
\widetilde{z}=\zeta\left(\lambda, P^{2}\right), \quad \widetilde{y}=\eta\left(\lambda, P^{2}\right)
$$

For any unipotential model it turns out that $N=\widetilde{y}^{2}+2 V$ is a constant of the motion; fixing $k^{\alpha}$ and the individual masses amounts to fix its numerical value, say $N=-\Lambda$, where

$$
\Lambda=\frac{M^{2}}{4}+\frac{\nu^{2}}{M^{2}}-\mu
$$

is intimately related to properties of relative motion 3 . The inverse formula

$$
M^{2}=2(\mu+\Lambda)+2 \sqrt{(\mu+\Lambda)^{2}-\nu^{2}}
$$

entails the development

$$
M=m_{1}+m_{2}+\frac{m_{0}}{2} \frac{\Lambda}{m_{0}^{2}}+O\left(\left(\frac{\Lambda}{m_{0}^{2}}\right)^{2}\right)
$$

\footnotetext{
${ }^{3}$ This quantity appeared, denoted as $b^{2}$ in the work of Todorov [2]
} 
hence $M \simeq m_{1}+m_{2}, M_{a} \simeq m_{a}$ in the nonrelativistic limit.

As proved in [17], in order to achieve a parametric description of the worldlines in terms of $\lambda$ we have to complete (34) with

$$
T=\left(\frac{M}{4}-\frac{\nu^{2}}{M^{3}}\right) \lambda+\frac{1}{M} \int F d \lambda-\frac{\nu}{M^{3}} \int G d \lambda
$$

where

$$
F\left(\lambda, M^{2}, \nu\right)=\{Q \cdot P, V\}, \quad G\left(\lambda, M^{2}, \nu\right)=\{z \cdot P, V\}
$$

Remark if $V$ doesnot depend on $y \cdot P$ then $G$ identically vanishes.

\section{Relative Motion}

Before proceeding further let us recall a few features of relative motion in nonrelativistic mechanics, for an isolated system of two point particles:

a) Relative motion is essentially the time evolution of the "radiusvector" joining the constituent bodies of the system. Obviously the values of this radius-vector are not points of an affine space but elements of a three-dimensional vector space; however it is natural to take the center of mass as origin of space, which leads to construct the positions of the relative particle moving "around it".

b) The motion of the center of mass gets completely separated from relative motion.

c) Equivalence: knowledge of the binary motion determines both the relative motion and the center-of-mass motion, and vice versa.

d) The mass of this ficticious particle, referred to as the reduced mass, is unambiguously determined from the individual masses of the constituent of the system, say $m_{0}=m_{1} m_{2} /\left(m_{1}+m_{2}\right)$, through the separation of center of mass from the relative degrees of freedom.

e) In the limit where the mass ratio $m_{1} / m_{2} \rightarrow 0$, the light body comes to coincide with the relative particle.

f) Relative motion is viewed as submitted to external forces, derived from an external potential. With the convention just made in (a) above, this potential can be seen as created by a ficticious source located at the center of mass.

g) Hamiltonian formulation holds as well for the binary system as for the relative particle, and the correspondance is ensured by well-known formulas.

Remark Note that the points (a)(b)(c) are strictly kinematic, in this sense that they do not refer to a canonical formulation. In contrast 
the reduced mass invoked in (d) rests on the separation of internal vs external degrees of freedom, in the Hamiltonian framework. Finally (f)(g) explicitly refer to the Hamiltonian formalism.

Remark. In nonrelativistic mechanics the concept of center of mass (once the masses of the bodies have been fixed) is of pure kinematics, we could almost say pure geometry. In contrast, in special relativity one runs into the problem of spacetime foliation; the way out is clear provided the binary system is isolated: linear and angular momenta are conserved; the former provides a preferred foliation and the latter can be combined with the former in formula (20) which yields the center-ofmass coordinates. The principles of this procedure have been discussed many years ago by Pryce [16] and by Moeller [20]. But some dynamics is already implicitly involved here.

Remark. In classical mechanics the relative particle is definitely the most simple effective one-body system equivalent to the binary system.

Now we return to special relativity, with canonical equations of motion (15). We aim at giving a clear definition of relative motion in spacetime. We cannot expect that all the features of the classical theory can be generalized easily. So, as a first step, we start with a purely kinematic construction of the relative motion.

\subsection{Kinematics, relative particle.}

In this subsection we provisionally forget our canonical equations of motion (15). A motion of two pointlike (structureless) bodies is essentially a pair of worldlines. At this stage we need not specify the dynamics, except for

Assumption A1 There exists a center of mass, with definite mass $M$ and constant momentum $k^{\alpha}$ (such that $k \cdot k=M^{2}$ ) moving along a (timelike) straight line.

Let $\Xi^{\alpha}$ be the center-of-mass coordinates; we can write

$$
\Xi^{\alpha}=\Xi_{(0)}^{\alpha}+T \frac{k^{\alpha}}{M}
$$

$\Xi_{(0)}$ being an origin taken on the center-of-mass worldline; automatically $T$ is the center-of-mass proper time. The rest frame of the system is determined by the direction of $k^{\alpha}$. Given $k^{\alpha}$ we have a distinguished slicing of spacetime by three-planes orthogonal to it. This allows for 
an equal-time description of motion, such that $x_{1}$ and $x_{2}$, hence also $r=x_{1}-x_{2}$, are functions of the center-of-mass time $T$, whereas

$$
x_{1}^{0}=x_{2}^{0}=T
$$

(this formula, stems from equal-time description, irrespective of how interaction is modelled).

The four-vector $r=x_{1}-x_{2}$ will be called be the radius-vector; it is spacelike and has the usual physical meaning when it is orthogonal to linear momentum $k$. By itself it defines no point in the (affine!) Minkowski space, but in the spirit of (a) listed above, Relative motion can be though of as that of a ficticious particle with position $x_{\mathrm{rel}}^{\alpha}$ defined by attaching the radius-vector to the center of mass, in other words the relative particle is unambiguously defined by

$$
x_{\mathrm{rel}}=\Xi+\widehat{r}
$$

The motion of the bodies will be described by the sequence of the couples $x_{1}, x_{2}$ taken on the worldlines and simultaneous with respect to the slicing of spacetime determined by $k^{\alpha}$.

Since we consider only simultaneous positions, $r$ is orthogonal to $k$, and in the rest frame we have $r_{0}=0$ and $x_{a}^{0}$ as in (27)

Separating time from space in (42) we get

$$
x_{\mathrm{rel}}^{0}=\Xi^{0}
$$

and $\widehat{x}_{\text {rel }}=\widehat{\Xi}+\widehat{r}$ which becomes, by a choice of the origin such that $\Xi_{(0)}^{\alpha}=0$,

$$
x_{\mathrm{rel}}^{i}=r^{i}
$$

But $\Xi^{0}$ is the center-of-mass time, thus in view of (18) equation (43) simply means

$$
x_{\text {rel }}^{0}=T
$$

Now the motion of the relative particle is defined by (44) (45). Projection of the relative worldline onto the three-dimensional plane $T=0$ may be trivially identified with the relative orbit.

So far we have constructed the relative particle as undergoing a ficticious one-body motion, characterized by its worldline; now conversely, given this worldline and that of the center of mass, is it possible to reconstruct the initial couple of worldlines, say $x_{1}\left(\tau_{1}\right), x_{2}\left(\tau_{2}\right)$ ? Only if the answer is yes, the relative-particle motion can be considered as equivalent to that of the binary object.

Notice also that, although the relative particle undergoes a perfectly well-defined motion, kinematics alone provide no hint about its energy 
or its mass; these quantities could be eventually exhibited if we were able of setting a one-body canonical formalism that pure kinematics ignore.

In fact we have elaborated in kinematic terms a correspondance from motion to motion.

In order to address the issue of equivalence (which regards the possibility of reconstruction of the worldlines) let us now be more specific and assume

Assumption A2 Considered at equal times, the center of mass is aligned with $x_{1}$ and $x_{2}$ and lies between them.

This statement may be expressed as follows

$$
\widehat{x}_{1}-\widehat{\Xi}=b \widehat{r}, \quad \widehat{x}_{2}-\widehat{\Xi}=-a \widehat{r}
$$

where $a$ and $b$ are positive functions of $T$ such that $a+b=1$.

It follows immediately that

$$
\widehat{\Xi}=a \widehat{x}_{1}+b \widehat{x}_{2}
$$

In order to re-construct the worldlines of the binary motion we are provided with (46) and the equal-time condition (41). The only truble is about the space part: though $\hat{\Xi}$ and $\hat{r}$ are given inputs, solving (46) for $\widehat{x}_{1}$ and $\widehat{x}_{2}$ still requires knowledge of the coefficients $a, b$. As obvious in (47) these coefficients are the ingredients of $\widehat{\Xi}$, but starting from $\Xi$ and $x_{\text {rel }}$, it is generally not possible to revert back to $a$ and $b$.

Remark In the most generic case, knowing also the individual masses $m_{1}, m_{2}$ would not help: $a-b$ is some dynamical variable of the binary motion, not always a constant 4 Further information about $a$ and $b$ requires specifying how is the center of mass defined within the underlying dynamics, which goes beyond the kinematic approach proposed in the present subsection, so

In purely kinematic terms, with center of mass and relative particle as the only data, re-constructing the worldlines of binary motion is generally not possible.

For an illustration, let us now focus on a two-body system described by the a priori Hamiltonian formalism of predictive relativistic mechanics, with help of the equal-time prescription; for the moment $U$ is possibly nonzero. Remember (24). Then formula (16) tells that at equal

\footnotetext{
${ }^{4}$ In the special case where $a-b$ is a constant of the motion, an alternative problem would consist in giving $a$ and $b$ instead of the individual masses; this problem is already and trivially solved by (A2); note that requiring constancy of $a-b$ is only a little more general than assuming a unipotential model.
} 
times we can write $\Xi=\frac{1}{2}\left(q_{1}+q_{2}\right)+\left(\frac{y \cdot P}{P^{2}}\right) z$. But then $q_{a}=x_{a}$, and $z=r$. Finally we get

$$
\begin{aligned}
& x_{1}=\Xi+\left(\frac{1}{2}-\frac{y \cdot P}{M^{2}}\right) r \\
& x_{2}=\Xi-\left(\frac{1}{2}+\frac{y \cdot P}{M^{2}}\right) r
\end{aligned}
$$

implying

$$
\begin{aligned}
& \widehat{x}_{1}=\widehat{\Xi}+\left(\frac{1}{2}-\frac{y \cdot P}{M^{2}}\right) \widehat{r} \\
& \widehat{x}_{2}=\widehat{\Xi}-\left(\frac{1}{2}+\frac{y \cdot P}{M^{2}}\right) \widehat{r}
\end{aligned}
$$

that is an example of formulas (46), where

$$
\frac{y \cdot P}{P^{2}}=\frac{a-b}{2}
$$

In these formulas $r^{\alpha}$ is given by the worldline of the "relative particle". Indeed this worldline can be parametrized with help of the center-ofmass time, say (44) (45) where $r^{i}$ takes on the form $r^{i}=\phi^{i}(T)$, more general than $\zeta^{i}$, since here $U$ is not yet assumed to be zero.

In contrast the quantity $y \cdot P$ is generally not fully determined by knowing relative motion and center-of-mass motion, because of the contribution of $U$ to it. Intuitively we could put it that way: $U$ carries dynamical information which goes beyond simple kinematics.

Naturally this difficulty disapears if we decide to focus on unipotential models, as we do in the sequel; indeed now we can write

$$
a=\frac{M_{1}}{M}, \quad b=\frac{M_{2}}{M}
$$

It follows that

$$
a-b=2 y \cdot P / M^{2}=2 \nu / M^{2}
$$

which is a constant and depends only on the masses $M, m_{1}, m_{2}$ (note that $a \leq b)$. So we can state

Theorem 1 For unipotential models, knowing the relative motion, its worldline and the mass of the center of mass, plus the squared-mass difference 5 , amounts to knkow both worldlines of the interacting bodies.

\footnotetext{
${ }^{5}$ This is a little more general than assuming that we know both individual masses.
} 
In the present case we can say that the one-body motion of the relative particle is equivalent to the binary motion.

We stressed in [17] the importance of having both individual energies positive, as we assume henceforth, which amounts to demand

$$
m_{1}^{2}+\Lambda>0
$$

Under this condition we proved

Proposition 2 If we can neglect $\sqrt{|\Lambda|}$ in front of $m_{2}$, then we have that, at equal times $\Xi$ and $x_{2}$ coincide in the limit $m_{1} / m_{2} \rightarrow 0$.

This statement was labelled as "theorem 2" in [17]. Since $\Xi$ and $x_{2}$ are coordinates of points describing timelike curves, their coincidence at equal times implies coincidence of the worldlines; this amounts to say that, taking $m_{1} / m_{2}$ to zero, the worldline of the center of mass coincides with that of the heavy particle. We define $\gamma=\frac{m_{1}}{m_{2}}$ and $\varepsilon=\gamma^{2}$.

In fact the assumption made about $\Lambda$ is superfluous for negative $\Lambda$, owing to (53) which requires $|\Lambda|<m_{1}^{2}=\varepsilon m_{2}^{2}$. So in this case we can write

$$
\Lambda=a m_{1}^{2}=a \varepsilon m_{2}^{2}
$$

where $|a|<1$. Although $\Lambda \rightarrow 0$ with $\varepsilon$, a glance at (14) shows that $\frac{|\Lambda|}{m_{0}^{2}}$ remains $>|a|$, so the situation is clearly distinct from a nonrelativistic regime.

In contrast for positive $\Lambda$ neglecting $\sqrt{|\Lambda|}$ in front of $m_{2}$ is essential. If we fix a priori $\Lambda$ independent from $\gamma$, the recoil of $x_{2}$ generally does not vanish when $\gamma \rightarrow 0$. In contrast taking $\Lambda=O\left(\gamma^{p}\right)$, for some positive power of $\gamma$, entails $M \rightarrow m_{2}, x_{2} \rightarrow \Xi$, etc (see 5 of [17]).

Vanishing $\Lambda$ is a trivial case.

With help of the above proposition (and under the same assumption) let us derive the following

Proposition 3 Neglecting $\sqrt{|\Lambda|}$ in front of $m_{2}$, we have that $x_{1} \rightarrow x_{\mathrm{rel}}$ when $m_{1} / m_{2} \rightarrow 0$, in other words the light body comes to coincide with the relative particle.

Proof We use the equal-time description. On the one hand definition (42) of $x_{\text {rel yields }}$

$$
\widehat{x}_{\mathrm{rel}}=\widehat{\Xi}-\widehat{x}_{2}+\widehat{x}_{1}
$$


but, Proposition 2 entails $\widehat{\Xi}-\widehat{x}_{2} \rightarrow 0$ hence $\widehat{x}_{1} \rightarrow \widehat{x}_{\text {rel }}$. On the other hand, working at equal times, formula (41) holds true; we also have have (45), therefore $x_{1}^{0}=x_{\text {rel }}^{0}=T$. Finally $x_{1} \rightarrow x_{\text {rel }}$. []

So properties c) and e) of the list in 2.3 are extended to the relativistic realm. In contrast extension of d) f) g) remain problematic for, in the kinematic context, there is no indication as to know whether the relative motion also can be derived from a Hamiltonian of its own. This question leads us to dynamical considerations.

Before that we turn to dynamics, let us summarize the equal-time description of the relative particle. On the one hand we have, for the spatial relative variables, the evolution equations (31) (32). On the other hand we have (45), but now the unipotential assumption entails that $T$ is given by (38). Defining $\check{E}$

$$
\breve{E}=\frac{M}{4}-\frac{\nu^{2}}{M^{3}}=\frac{\left(P \cdot p_{1}\right)\left(P \cdot p_{2}\right)}{M^{3}}=\frac{M_{1} M_{2}}{M}
$$

(note that $\breve{E}$ cannot be negative) we re-write (38) as follows

$$
T=T(\lambda)=\breve{E} \lambda+\frac{1}{M} \int F d \lambda-\frac{\nu}{M^{3}} \int G d \lambda
$$

According to (36) and using $r^{i}=z^{i}$ (equal times), we have

$$
x_{\mathrm{rel}}^{i}=\zeta^{i}\left(\lambda, M^{2}, y \cdot P\right)
$$

where $\zeta^{i}$ are three functions defined in (34) as solutions of (31) (32). Moreover by (45)

$$
x_{\mathrm{rel}}^{0}=T(\lambda)
$$

In the context of our present assumptions, equations (56) (57) characterize the equal-time description of the relative particle.

Although we considered a Hamiltonian model of the binary object, the motion of our relative particle is not derived from any canonical formalism. At this stage there is no indication about its mass 6 , in other words the concept of a reduced mass is still lacking.

\subsection{Dynamics, effective particle.}

In the previous subsection devoted to kinematics we were interested in two-body and one-body motions. Here we come to dynamical systems,

\footnotetext{
${ }^{6}$ In the absence of a canonical formulation, enforcing $m=m_{0}$ would not be here justified as much as it is in the nonrelativistic domain.
} 
keeping clear in mind that a system essentially is a collection of possible motions.

Now we consider an alternative approach to the problem of reducing binary motion to a one-body problem.

In a more general setting, what we search now is a ficticious one-body dynamical system of which the solution gives knowledge of the binary motion; in the most simple and the most reasonable manner. We mean a canonical formulation of the kind displayed in Section 2.1.

More precisely, for any fixed value $k^{\alpha}$ of linear momentum, and setting $k \cdot k=M^{2}$, we look for a one-body Hamiltonian $H$, depending on $k^{\alpha}$ as a parameter,such that it generates the motion of a ficticious particle referred to as effective. To be more specific we require that the external field (involved in the motion of the ficticious particle) is stationary, and that the unit vector $u$ present in Section 2.1 is just $k / M$. This amounts to identify $\widehat{\delta}$ of (23) with $\delta_{\perp}$ of 2.1 , and $\widehat{x}$ with $x_{\perp}$. Naturally we intent to remain as much as possible in the spirit of what is usually done in Newtonian mechanics, so we aim at a relativistic extension of several (if not all) points of the list a)-g). We are not expecting to fulfill the whole list, for example reference to the vectorradius (point a) is not a priori required, though welcomed when possible.

For simplicity we assume from now on that $V$ doesnot depend on $y \cdot P$. It follows that

$$
V=V\left(\widetilde{z}^{2}, \widetilde{y}^{2}, \widetilde{z} \cdot \widetilde{y}, P^{2}\right)
$$

This restriction entails that $G$ and $\partial W / \partial p_{0}$ identically vanish; formula (38) reduces to

$$
T(\lambda)=\breve{E} \lambda+\frac{1}{M} \int F d \lambda
$$

A clue toward a canonical one-body formalism is provided by the striking similarity of (31) (32) with (44) (15), that we pointed out several decades ago [19]. Actually they are the same formulas, up to notation. The Lie algebras generated by $\widetilde{z}^{\alpha}, \widetilde{y}^{\beta}, P^{\gamma}$ under the Poisson brackets $\{. ., .$.$\} and by x_{\perp}^{\alpha}, p_{\perp}^{\beta}, M u^{\gamma}$ under the brackets $(. ., .$.$) are manifestly iso-$ morphic, for instance compare $\{\widetilde{z}, \widetilde{y}\}=\widehat{\delta}$ with $\left(x_{\perp}^{\alpha}, p_{\perp}^{\beta}\right)=\delta_{\perp}$. Since we have the same Poisson bracket structure, it is clear that from (31) (32) we obtain (4) (5) provided we replace

$$
\lambda \mapsto \tau, \quad \widetilde{z} \mapsto x_{\perp}, \quad \widetilde{y} \mapsto p_{\perp}, \quad V \mapsto W
$$

where

$$
W=\operatorname{subs} .\left(V \mid \widetilde{z}=x_{\perp}, \widetilde{y}=p_{\perp}, P^{\alpha}=M u^{\alpha}\right)
$$


The substitution above introduces no dependence on $x^{0}$ for $W$, which is automatically constructed as stationary and spherically symmetric.

The observation presented in (60) (61) makes the two-body problem equivalent to a one-body problem, at least in sofar as space relative variables $\widetilde{z}, \widetilde{y}$ are concerned.

Let us try to go one step further. Since equations (4) (5) are just $a$ subset of the system (2) generated by $H=\frac{1}{2} p^{2}+W$, it is natural to investigate as to know to which extent the full binary dynamics can be reduced to that of the one-body Hamiltonian system described by all the equations (2) with $W$ defined in (61). To this end we introduce a ficticious pointlike body moving in the external interaction potential $W$. This system, governed by the Hamiltonian generator $H$, will be referred to as the effective particle. Let $x_{\text {eff }}^{\alpha}$ be its coordinates.

Remark $\mathrm{W}$ depends on $k^{\alpha}$ as a parameter; to each binary subsystem characterized by fixing $P^{\alpha}=k^{\alpha}$ corresponds a distinct one-body dynamical system. Formula (61) uniquely maps a system of equations (ruling the dynamical subsystem corresponding to a choice of $k^{\alpha}$ ) to another system of equations (deduced from $H$ and ruling the effective particle). But their solutions are still to be specified by initial conditions (for instance fixing the numerical values of first integrals). As a result the correspondance between pairs of worldlines and one-body motions might be affected by some arbitrariness. Nevertheless to each solution $\widetilde{z}=\zeta\left(\lambda, P^{2}\right), \widetilde{y}=\eta\left(\lambda, P^{2}\right) \quad$ of (31) (32) we can associate

$$
x_{\perp}=\zeta\left(\tau, M^{2}\right), \quad p_{\perp}=\eta\left(\tau, M^{2}\right)
$$

which is a solution of (4) (15).

It will be useful to distinguish, among two-body phase space functions, those that are of the form $J=\mathcal{J}(\widetilde{z}, \widetilde{y}, P)$. Let us call them functions of the special type. As an example $\widetilde{y}^{2}, V, N$ are of the special type; in contrast $y \cdot P$ is not .

We trivially extend to special-type functions the substitution (61) carried out in $V$, and write

$$
\text { subs. } J=\mathcal{J}\left(x_{\perp}, p_{\perp}, k\right)
$$

Let $I=\mathcal{I}(\widetilde{z}, \widetilde{y}, P)$ be any first integral of the special type. Inserting (34) into $\mathcal{I}$ we compute and find a result depending on the functions $\zeta, \eta$ chosen among possible solutions, but independent of $\lambda$, say $C$. The one-body conterpart of $I$ is subs. $I=\mathcal{I}\left(x_{\perp}, p_{\perp}, k\right)$. Inserting (62) into subs. $I$ we automatically get a result independent of $\tau$ which is the same number $C$ (see Appendix 1 for an example). Thus 
Proposition 4 When fixing the numerical value of a first integral of the special type, we assign the same value to its one-body conterpart.

This remark can be applied to $L^{\alpha}$. Thus setting $L^{\alpha}=l^{\alpha}$ implies

$$
\varepsilon_{\alpha \mu \nu \rho} k^{\mu} x_{\perp}^{\nu} p_{\perp}^{\rho}=l_{\alpha}
$$

ensuring that the orbit of the binary motion and that of $x_{\text {eff }}$ lay in the same two-dimensional plane.

Application to $N$ imposes the same numerical values to $N$ and to $p_{\perp}^{2}+$ $2 W$. But one-body Hamiltonian mechanics yields (11). Hence

$$
E^{2}-m^{2}=\Lambda
$$

where now $m$ is the mass of the effective particle, we shall refer to it as the reduced mass. This formula obviously requires

$$
m^{2}+\Lambda>0
$$

\subsubsection{Motion of the effective particle.}

As already noted previously, the space part of its equations of motion is ruled by (44) (51) analogous to (31) (32). Applying substitution (61) to (34) we obtain the solutions

$$
x_{\mathrm{eff}}^{i}=\zeta^{i}\left(\tau, M^{2}\right), \quad p_{\mathrm{eff}}^{i}=\eta^{i}\left(\tau, M^{2}\right)
$$

where $\zeta^{i}$ and $\eta^{j}$ are the functions defined in (34) as solutions of (31) (32). The first formula in (66) is reminiscent of (56) but should not be confused with it, because $\tau$ may be different from $\lambda$.

Now consider the time part of the equations of motion.

On the one hand remember that our splitting of spacetime refers to the center-of-mass frame; thus we must identify the coordinate time of the effective particle with the time of the center of mass, like in formula (45), in other words

$$
x_{\mathrm{eff}}^{0}=x_{\mathrm{rel}}^{0}=T
$$

whith $T$ already given by (38), where now $G=0$ and $F$ is a function of the special type.

On the other hand integrating the canonical equations of motion yields formula (10), where now $\partial W / \partial p_{0}$ vanishes thus

$$
x_{\mathrm{eff}}^{0}=E \tau+\text { const. }
$$


and $E$ is necessarily positive.

Comparing (67) with (68) and taking (59) into account yields this relation $E \tau=\breve{E} \lambda+\frac{1}{M} \int F d \lambda+$ const. This formula cannot yet completely define $\tau$ as a function of $\lambda$ (or vice versa), for two reasons: on the one hand the arbitrary integration constant involved in the indefinite integral should be fixed; on the other hand $E$ remains to be specified. First it is natural to demand, as a defining rule

$$
E \tau=\breve{E} \lambda+\frac{1}{M} \int_{0}^{\lambda} F d \lambda
$$

indeed when $F$ is a constant of the motion it allows trivially to identify $\tau$ with $\lambda$.

Then choosing $E$ in terms of the two-body constants of motion will determine a unique map from the binary pairs of worldlines to the motions of $x_{\text {eff }}$, or equivalently (by Theorem 1 ) a map from the worldlines of $x_{\text {rel }}$ to those of $x_{\text {eff }}$. We say that each choice of this kind produces a version of the effective particle. Naturally our freedom about $E$ will be constrained by obvious restrictions. For instance according to (64), each choice of $E$ implies a unique expression for $m$ (and conversely); it is clear that this $m$ must tend to $m_{0}$ in the nonrelativistic limit.

For practical purpose we sometime prefer discussing the choice of $m$, which is the relativistic reduced mass, and then derive the corresponding value of $E$.

Now a relevant question is asking to which extent the effective particle can coincide with the relative particle defined by (42). Indeed (according to Theorem 1 above) in such a case the effective-particle motion would encode all information about the binary motion.

So let us compare effective and relative particles; their time parts are

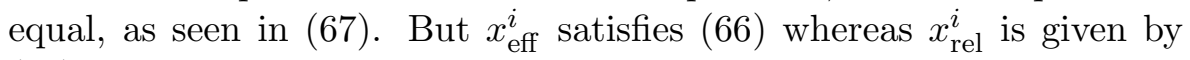
(56). Appearance of the same functions $\zeta$ ensures that effective and relative particles have the same orbit in the vector space orthogonal to $k^{\alpha}$. However the worldlines are generally different due to equation (69) which makes $\tau$ generally distinct from $\lambda$, implying that space and time coordinates are differently related in $x_{\text {eff }}$ and in $x_{\text {rel }}$. In other words the schedules do not generally coincide; actually they coincide iff $\tau=\lambda$, which is possible by a suitable choice of $E$ provided that the right-hand side of (69) is linear in $\lambda$. Let us summarize as follows

Theorem 2 Effective particle and relative particle have the same orbit, but in general they have different schedules. They have the same worldline iff $\tau=\lambda$, which is not always possible. 
Unfortunately, requiring equality of $\tau$ with $\lambda$ is a very restrictive condition. It can be satisfied for all motions of the system when $F$ is a first integral, provided we choose

$$
E=\breve{E}+\frac{F}{M}
$$

Alternatively it could be satisfied without restrictions about $F$ but only for circular motions (this last point stems from the fact that $F$ remains constant on any circular orbit, as pointed out in proposition 5 of [17]). An example is given in Appendix 2.

Having $F$ constant for all motions is rather exceptional; in particular it is satisfied when $\partial V / \partial P^{2}=0$. In contrast circular motions exist under very large assumptions (see [17] ). We can state

Theorem 3 In the academic case where $F$ is a first integral of the binary system, the substitution (61) together with identifications $\tau=\lambda$ and $E$ chosen as in (70) above makes the worldline derived from $H=$ $\frac{1}{2} p^{2}+2 W$ to coincide, for all motions of the system, with that of the relative particle defined in kinematical terms through (44) (45).

However one must realize that in most realistic systems the assumptions of Theorem 3 are not satisfied. In the most general situation we are left with (69) which implicitly defines $\tau$ as a function of $\lambda$ ( or vice versa) but this function can be complicated.

Thus in general it is not possible to demand that $x_{\mathrm{eff}}$ and $x_{\mathrm{rel}}$ coincide.

Equivalence.

We saw previously (Theorem 1 ) how the relative particle can be considered as equivalent to the binary system.

In order, for the effective particle, to deserve its name, the question is whether binary motion can be similarly reduced to that of the effective particle; in other words: is it possible to reconstruct the two-body worldlines just by taking the effective particle motion (and center of mass) as input?

Since we are dealing with unipotential models we are already sure by Theorem 1 that relative motion encodes the worldlines of the two-body system. Thus in order to check the reconstruction property of effective motion it is sufficient to observe that

Proposition 5 Once the effective particle's version has been choosen, knowledge of $x_{\mathrm{eff}}$ entails knowledge of $x_{\mathrm{rel}}$. 
Proof The choice of a version means fixing $E$ in terms of binary first integrals. Substitution (61) is manifestly invertible so we know the functions $V$ and $W$ defined on their respective phase spaces. Relative and effective particles have the same orbit. All we still need is a one-toone correspondance between their schedules; fortunately formula (69) yields $\tau$ as a function of $\lambda$ as well as (implicitly) the reverse. []

\subsubsection{Choosing a version}

If we leave aside the academic case presented in Theorem 3, no choice of $E$ would make effective particle and relative particle identical. Still we may look for a "good choice" motivated by some reasonable requirement or by the sake of simplicity; in any case $m$ should coincide with $m_{0}$ in the nonrelativistic limit. This remark will not yet select a unique version of the effective particle, therefore it is convenient to sketch a few possibilities (among others):

i) The most simple choice seems to consist in defining the reduced mass by the conventional formula used in nonrelativistic mechanics, say $m=m_{0}$. According to (64) and (35) this implies

$$
E^{2}=m_{0}^{2}+\frac{M^{2}}{4}+\frac{\left(m_{1}^{2}-m_{2}^{2}\right)^{2}}{4 M^{2}}-\frac{1}{2}\left(m_{1}^{2}+m_{2}^{2}\right)
$$

Since $m_{0}<m_{1} \leq m_{2}$, for negative $\Lambda$ choosing $m=m_{0}$ is submitted to the condition $m_{0}^{2}+\Lambda \geq 0$, more restrictive than (53).

ii) Alternatively we can adopt the definition postulated by Todorov 2 many years ago, $m=m_{T}=\frac{m_{1} m_{2}}{M}$. In our notation (13) we can write $m_{T}^{2}=\frac{\mu^{2}-\nu^{2}}{M^{2}}$. Then (64) and (35) entail

$$
E^{2}=\frac{1}{4 M^{2}}\left(4 \mu^{2}+M^{4}-4 \mu M^{2}\right)=\frac{1}{4 M^{2}}\left(M^{2}-2 \mu\right)^{2}
$$

Since $\frac{\partial W}{\partial p_{0}}$ is zero, $\mathrm{E}$ cannot be negative,

$$
E=\frac{1}{2 M}\left|M^{2}-\left(m_{1}^{2}+m_{2}^{2}\right)\right|
$$

which re-discovers the expression found in [2] for the energy of the effective particle. It is well-known that $m_{T} \rightarrow m_{0}$ in the nonrelativistic limit.

iii) Another version, inspired by relativistic quantum mechanics, may be considered by demanding that, in case of bounded motion, the "unrest energy" of the effective particle, we mean $E-m$, be strictly 
equal to the binding energy of the binary system, namely $M_{\text {bin }}=M-$ $\left(m_{1}+m_{2}\right)$. Then we get

$$
2 m=\frac{\Lambda}{M_{\text {bin }}}-M_{\text {bin }}
$$

which implies that $\Lambda-M_{\text {bin }}^{2}$ must have the sign of $M_{\text {bin. }}$. Then the development (37) yields

$$
\frac{\Lambda}{M_{\mathrm{bin}}}=2 m_{0}+O\left(\frac{\Lambda}{m_{0}^{2}}\right)
$$

hence $m=m_{0}+O\left(\frac{\Lambda}{m_{0}^{2}}\right)$, which reduces to $m_{0}$ in the nonrelativistic limit, as it should.

iv) We could also try choosing $E$ such that $\tau=\lambda$ on each circular orbit; this can be actually carried out for a toy model given in Appendix 2 .

\section{Conclusion}

Relative motion is a natural concept of geometrical origin, basically founded on evolution of the radius-vector. But defining the relative particle requires a previous definition of center of mass. Under this condition the relative particle is always well-defined in geometrical terms, without specifying in more details how interaction is described between the two bodies. Assumptions A1 and A2, being sufficiently general, are likely to accomodate a large number of theories. In principle, this (almost) purely kinematic approach is very general; but it would remain academic unless we address the issue of equivalence. Considering this question in the general framework of predictive relativistic dynamics, we realized that the map of the binary motion onto that of a single particle is not always unambiguously invertible: some dynamical information (the function $U$ ) is needed in order to reconstruct the worldlines of the binary object from the motion of the relative particle.

This limitation led us to focus on unipotential models of mutual interaction, and in this context it was indeed possible to derive some nice properties of the relative particle (Theorem 1 and Proposition 3) satisfying the points c) and e) of the list in Section 3. However some features of the classical theory, namely the reduced mass and the canonical formalism, had no relativistic conterpart at this stage. 
This shortcoming was calling for an alternative approach; therefore we proposed that beside the relative particle one considers another ficticious body, referred to as effective, which is, by a simple rule, constructed as a Hamiltonian system. This procedure involves some arbitrariness related to the ajustment of a constant of the motion, which can be interpreted as some freedom in the choice of the relativistic reduced mass. One of the possible choices retrieves Todorov's effective particle originated from QED [2] and further given a worldline content [3] in the framework of relativistic constraint dynamics.

The relative particle is unambiguously defined whereas the effective particle is a priori affected by an arbitrariness, the solution of which we had to discuss. Fortunately it turns out that relative and effective particle have anyway the same orbit and differ only by their schedules; only in particular cases they completely coincide. So in practice various versions of the effective particle are more or less equally useful; further investigation might bring out a preferrence among the possible choices just listed above (a list which is not exhaustive).

Most part of our picture has been elaborated in the context of rather simple hypotheses; more investigation is needed for instance if we relax the assumption that $\partial V / \partial(y \cdot P)$ vanishes. But we hope that the present work will already clarify several ideas about relative motion in Minkowski spacetime.

\section{Appendix 1}

Consider the toy model presented in [17], say

$$
V=\chi \sqrt{P^{2}} \widetilde{z}^{2}
$$

with $\chi$ a positive string constant. Fixing $P^{\alpha}=k^{\alpha}$ and fixing $L^{\alpha}=l^{\alpha}$ orthogonal to it, the solution to (31) (32) is

$$
\begin{gathered}
\widetilde{z}=\zeta(\lambda)=A \sin (\Omega \lambda+\Phi)+B \cos (\Omega \lambda+\Phi) \\
\widetilde{y}=\eta(\lambda)=A \Omega \cos (\Omega \lambda+\Phi)-B \Omega \sin (\Omega \lambda+\Phi)
\end{gathered}
$$

The orbital plane is orthogonal to both $k$ and $l$, and $A, B$ are mutually orthogonal spacelike constant vectors in that plane $(|A|$ and $|B|$ are the half-axes of an ellipse). $\Phi$ is a scalar constant, moreover we have $\Omega=\sqrt{2 \chi M}$. Consider the first integral $N=\mathcal{N}(\widetilde{z}, \widetilde{y}, P)=\widetilde{y}^{2}+2 V$. From (73) (74) we compute $\widetilde{z}^{2}$ and $\widetilde{y}^{2}$, hence we find that the numerical value of $N$, say

$$
<N>=2 \chi M\left(A^{2}+B^{2}\right)
$$


is (as expected) independent of $\lambda$.

In the substitution (60) (61) the one-body conterpart of $V$ is $W=$ $\chi M x_{\perp}^{2}$ and the conterpart of $N$ is

$$
\operatorname{subs.} N=\mathcal{N}\left(x_{\perp}, p_{\perp}, k\right)=p_{\perp}^{2}+2 \chi M x_{\perp}^{2}
$$

To the solution (73) (174) of the binary problem we associate

$$
\begin{gathered}
x_{\perp}=\zeta(\tau)=A \sin (\Omega \tau+\Phi)+B \cos (\Omega \tau+\Phi) \\
p_{\perp}=\eta(\tau)=A \Omega \cos (\Omega \tau+\Phi)-B \Omega \sin (\Omega \tau+\Phi)
\end{gathered}
$$

Inserting (76) (77) into (75) yields of course

$$
p_{\perp}^{2}+2 \chi M x_{\perp}^{2}=2 \chi M\left(A^{2}+B^{2}\right)
$$

manifestly independent of $\tau$ and identical to $\langle N\rangle$.

\section{Appendix 2}

For the same model, beside $\Lambda$ we have another first integral $j^{2}$ given by (33), and

$$
\frac{1}{2}\left(A^{2}+B^{2}\right)=-\frac{\Lambda}{4 \chi M}=\frac{<N>}{4 \chi M}, \quad A^{2} B^{2}=\frac{j^{2}}{\Omega^{2}}
$$

Straightforward calculations yield ([17])

$$
F=\chi M\left(A^{2} \sin ^{2}(\Omega \lambda+\Phi)+B^{2} \cos ^{2}(\Omega \lambda+\Phi)\right)
$$

a primitive of this function is

$$
\int_{0}^{\lambda} F d \lambda=\chi M\left(\frac{A^{2}+B^{2}}{2} \lambda+\frac{B^{2}-A^{2}}{4 \Omega} \sin (2 \Omega \lambda+2 \Phi)+\frac{A^{2}-B^{2}}{4 \Omega} \sin (2 \Phi)\right)
$$

Circular orbits are characterized by $A^{2}=B^{2}$, so if we define $F_{\text {cir }}=\frac{N}{4}$, this quantity, defined on the whole phase space, is a first integral which coincides with $F$ on any circular orbit.

Thus if we choose (for all orbits)

$$
E=\breve{E}+\frac{F_{\text {cir }}}{M}=\breve{E}+\frac{N}{4 M}
$$

equation (69) allows for having that $\tau=\lambda$ on any circular orbit. 


\section{References}

[1] E. Breźin, C. Itzykson, J. Zinn-Justin, Phys. Rev. D 1, 2349 (1970).

[2] I.T. Todorov, Quasi-potential approach to the two-body problem in quantum field theory, in "Properties of fundamental interactions", 9 part C, A. Zichichi Ed. Editrice Compositori, Bologna (1973).

V.A. Rizov, I.T. Todorov, B. L. Aneva, Nucl. Phys. B 98, 447-471 (1975).

[3] I.T. Todorov, JINR Report E2-10125, unpublished (1976).

V.V. Molotkov and I.T. Todorov, Commun. Math. Phys. 79, 111$132(1981)$

[4] P.A.M. Dirac, Canad. J. Math. 2, 129 (1950); Proc. Roy. Soc. A 246, 326 (1958).

[5] D. Alba, H. Crater, L. Lusanna, Jour. of Phys. A 40, 9585 (2007)

[6] A. Maheshwari, E.R. Nissimov, I.T. Todorov, Lett. in Math. Phys. Phys. Rev. D 5, 359 (1981).

[7] A. Buonanno, T. Damour, Phys. Rev. D 59, 084006 (1999).

[8] P.P. Fiziev, I.T. Todorov, Phys. Rev. D 63, 104007 (2001).

[9] S.B. Faruque, Fizika B 13, 699-710 (2004).

[10] Ph. Droz-Vincent, Reports in Math. Phys. 8, 79 (1975)

[11] Ph. Droz-Vincent, Ann. Inst. H. Poincaré, 27, 407 (1977)

[12] Ph. Droz-Vincent, Lett. Nuov. Cim. 1839 (1969); Physica Scripta 2, 120 (1970)

[13] L. Bel, Ann. Inst. Henri Poincaré, 12 , 307 (1970).

R. Arens, Arch. for Rat. Mech. and Analysis, 47 , 255 (1972).

[14] L. Lusanna, Il Nuov. Cim. 65 B , 135 (1981).

[15] Ph. Droz-Vincent, Jour. Math. Phys. 37, 4274-4291 (1996)

[16] M.H.L. Pryce, Proc. Roy. Soc. 195 A, 62 (1948)

[17] Ph. Droz-Vincent, Int. Jour. Theor. Phys. 50, 3481-3502 (2011).

[18] D.G. Currie, Journ. Math. Phys. 4, 1470 (1963), Phys.Rev. 142, 817 (1966). D.G. Currie, T.F. Jordan, E.C.G. Sudarshan, Rev.Mod.Phys. 35, 350 (1963).

[19] Ph. Droz-Vincent, C. R. Acad. Sciences, Paris 290, 115 (1980)

[20] C. Moeller Ann. Inst. Henri Poincaré, 11, 251 (1949). 